\title{
Statistical properties of random scattering matrices
}

\author{
Petr Šeba ${ }^{1}$, Karol Życzkowski ${ }^{2}$, and Jakub Zakrzewski ${ }^{2,3}$ \\ 1 Nuclear Physics Institute, Czech Academy of Sciences, \\ 25068 Rež near Prague, Czech Republik \\ ${ }^{2}$ Instytut Fizyki Mariana Smoluchowskiego, Uniwersytet Jagielloński, \\ ul. Reymonta 4, 30-059 Kraków, Poland \\ ${ }^{3}$ Laboratoire Kastler-Brossel, Université Pierre et Marie Curie, \\ T12, E1, 4 place Jussieu, 75272 Paris Cedex 05, France
}

(November 4, 2018)

\begin{abstract}
We discuss the properties of eigenphases of $S$-matrices in random models simulating classically chaotic scattering. The energy dependence of the eigenphases is investigated and the corresponding velocity and curvature distributions are obtained both theoretically and numerically. A simple formula describing the velocity distribution (and hence the distribution of the Wigner time delay) is derived, which is capable to explain the algebraic tail of the time delay distribution observed recently in microwave experiments. A dependence of the eigenphases on other external parameters is also discussed. We show that in the semiclassical limit (large number of channels) the curvature distribution of $S$-matrix eigenphases is the same as that corresponding to the curvature distribution of the underlying Hamiltonian and is given by the generalized Cauchy distribution.
\end{abstract}

72.20.Dp, 05.45+b,72.10.Bg. 


\section{INTRODUCTION}

Quantum chaotic scattering has been discussed for a number of years [1,2]. It may occur in a variety of different physical situations from atoms and nuclei to disordered mesoscopic devices or microwave cavities. The schematic model of the system is presented in Fig.11. The cavity (internal region) is coupled to the outside world by leads. The characterictics of the internal motion manifest themselves obviously in the properties of the $S$-matrix. This is seen directly using the Hamiltonian approach to the scattering [3].

Consider a simple Hamiltonian with the Hilbert space spanned by $N$ discrete states $\mid k>$ and $M$ continua $\mid c, E>$ :

$$
\begin{aligned}
H & =\sum_{k=1}^{N} E_{k}|k><k|+\sum_{c=1}^{M} \int d E E|c, E><c, E|+ \\
& +g \sum_{c=1}^{M} \sum_{k=1}^{N} \int d E\left(W_{k c}(E)|k><c, E|+\text { h.c. }\right) .
\end{aligned}
$$

Note that no continuum-continuum coupling is permitted in the model. The boundcontinuum coupling is characterized by coupling constant $g$ and the energy dependent matrix $W_{k c}(E)$, where it is assumed that the columns of $W$ are normalized to unity. If $W$ depends only weakly on the energy, the corresponding unitary $M \times M$ matrix $S$ may be expressed as [3, 4 ]

$$
S_{c c^{\prime}}(E)=\delta_{c c^{\prime}}-2 g^{2} i \sum_{k l} W_{c k}(E)\left(\frac{1}{E-\mathcal{H}}\right)_{k l} W_{l c^{\prime}}(E),
$$

where $\mathcal{H}$ is the effective Hamiltonian describing the motion within the bound subspace after

eliminating the continua in a Markov approximation. In an arbitrary basis spanning the bound subspace it takes the form

$$
\mathcal{H}_{k l}=H_{k l}-i g^{2} \sum_{c=1}^{M} W_{k c} W_{c l}
$$

The question may be posed whether, for generic systems, there is a unique relation between S-matrix properties and the type of motion inside the cavity. One way to address this 
issue is via the semiclassical theory [1] which seems, however, to be limited to a large number of channels, $M$. On the other hand, in recent experiments on scattering in microstructures [5], [6] $M$ can be of the order of unity.

A second possible way is a stochastic approach in which the Hamiltonian $H$ and the coupling matrix $W$ [2] are modeled by random matrices. A subsequent averaging over different realizations of $H$ (typically for fixed $W$ ) yields statistical predictions concerning fluctuations of physical quantities of interest. One hopes, extending the conjecture which has been quite useful for bounded chaotic systems [7] that the properties of fluctuations are universal. For bounded, autonomous, classically chaotic systems, depending on their symmetries, the statistical spectral fluctuations are, generically, well represented by the corresponding quantities obtained from Gaussian Orthogonal (GOE), Unitary (GUE), or Symplectic (GSE) Ensembles of random matrices [8].

Assuming that $H$ matrices are drawn from one of these ensembles and making similar assumptions on the coupling matrix $W$ one is forced to ask what are the properties of the ensemble of $S$-matrices. A partial answer has been obtained by Lewenkopf and Weidenmüller [2]: if $H$ belongs to GOE and the channels are equivalent (see below) $S$ matrix belongs to COE provided the coupling constant $g$ equals to unity. This result has been recently generalized to all three universality classes by Brouwer [9] for arbitrary $g$. Brouwer found that the $S$-matrices, Eq.(1.2), conform with Generalized Orthogonal (GCOE) and Unitary (GCUE) Circular Ensembles [10] which reduce to circular ensembles of Dyson (COE, CUE), [11] when the coupling to the continuum becomes ideal.

Brouwer's result provides a direct link with another popular, random matrix theory based approach in which one draws directly $S$-matrices, or transmission matrices, from an appropriate random matrix ensemble [10,12, 13]. The Hamiltonian approach is, in a sense, more general since it allows for calculation of time delays and energy-correlation averages, while the ensemble of $S$-matrices is energy independent. On the other hand, one is frequently interested in single-point with respect to energy statistical measures. Those may be directly accessed from the ensemble of $S$ matrices. In such a way one may obtain, e.g., the universal 
conductance fluctuations [14] from the random matrix model [12].

The study of mesoscopic devices points out that random matrix approach has its limitations, e.g., the fluctuations may become dependent on the length of the device [15]. It is still, an open question, what are the limits of the universality. We do not adress this problem here, rather we want to study, within the random model, the generic properties of $S$ matrices dependent on some parameter. In this way we extend recent intensive studies of the statistical properties of bound systems dependent on the external parameters [16 19].

For parameter independent Hamiltonians statistical properties are generically universal once the mean level spacing, $\Delta$ is known. Parametric measures reveal a similar universality, in addition to unfolding the energy levels one has to unfold the parameter dependence [16,17]. On the other hand, much less is known about the parametric behaviour of scattering systems. The two-point correlation function for the $S$ matrix with respect to an external parameter was derived recently by Macêdo [20]. The conductance fluctuations in the presence of the magnetic field induced time-reversal symmetry breaking have been studied by Pluhař et al. [21]. The semiclassical properties of the so called Wigner time delay have been discussed by Jalabert and Pichard [15]. Obviously, even in the absence of the external parameter $S$ matrix is energy dependent - the corresponding correlation function was obtained in [2].

Instead of investigating the $S$-matrix elements we focus on the properties of the corresponding eigenphases (phase shifts). Some important dynamical features of the system (for instance the time delay inside the interaction region) can be easily expressed using the derivatives of $S$-matrix eigenphases. Being directly accesible in experiments, statistical properties of phaseshifts deserve a detailed study. The nearest-neighbour spacing distribution has been discussed in Ref. [22,23]. As mentioned above we consider here parametric dependence of phaseshifts.

The paper is organized as follows. The analytic results concerning the distribution of velocities (i.e., first derivatives of eigenphases with respect to energy or other external parameter), also referred to as slopes, are discussed in Section II. Here we discuss also briefly the distribution of the second derivatives, i.e., the curvatures, of the eigenphases. These predic- 
tions are tested against numerical results obtained from simulations based on Eqs.(1.1-1.2) (and their generalization allowing for the presence of some external parameter) in Section III. For systems dependent on the external parameter one may also construct directly circular ensembles of scattering matrices. The corresponding results are presented in Section IV.

\section{PARAMETRIC HAMMILTONIAN APPROACH TO $S$ MATRIX}

Consider the unitary matrix $S$ defined by equations (1.2) and (1.3). To discuss properties of the eigenphases of the $S$ matrix it is convenient to rewrite Eq.(1.2) as

$$
S(E)=\frac{1+i A}{1-i A},
$$

with $A$ given by

$$
A=g^{2} W^{+} \frac{1}{E-H} W
$$

Eigenphases $s_{m}$ of the unitary matrix $S$ are related to eigenvalues $a_{m}$ of the $M \times M$ Hermitian matrix $A$ by [9]

$$
s_{m}=2 \arctan \left(a_{m}\right) \quad m=1, \ldots, M .
$$

Since $s_{m}$ is a function of $a_{m}$ the statistical properties of $s_{m}$ and $a_{m}$ are identical after unfolding. In the semiclassical limit (large $M$ ) it has been shown [9] that $A$ belongs to the same ensemble as $H$. This implies that the statistical properties (level spacing, number variance etc.) of $s_{m}$ are identical with those of the eigenvalues of $H$. Moreover, the relation (2.3) is useful when discussing the parametric dependence of $s_{m}$.

To see how it works consider the slopes of the eigenphases with respect to the energy $E$. They have a direct physical significance as time delays associated with the corresponding phaseshifts (see, e.g. [15]) while the average slope is just the celebrated Wigner time delay.

The eigenequation for $a_{m}$ : 


$$
A\left|f_{m}>=g^{2} W^{+} \frac{1}{E-H} W\right| f_{m}>=a_{m} \mid f_{m}>
$$

is equivalent to

$$
\left(H+\frac{g^{2}}{a_{m}} W W^{+}\right)\left|h_{m}>=E\right| h_{m}>,
$$

with $\mid f_{m}>$ and $\mid h_{m}>$ related by $\left|h_{m}>=\frac{1}{E-H} W\right| f_{m}>$. Note that the $N \times N$ eigenvalue problem Eq.(2.5) has, for fixed $E$, only $M$ nontrivial solutions $a_{m}$ and corresponding eigenvectors $\left|h_{m}\right\rangle$. This is related to the fact that $M-N$ eigenvalues of $W W^{+}$vanish. Differentiating Eq.(2.5) with respect to $E$ for nontrivial $a_{m}$ one gets

$$
\frac{g^{2}}{a_{m}^{2}} \frac{d a_{m}}{d E}<h_{m}\left|W W^{+}\right| h_{m}>=<h_{m} \mid h_{m}>.
$$

We shall assume from now on that $\mid h_{m}>$ are normalized to unity.

Using the relation (2.3) between $s_{m}$ and $a_{m}$, we express the inverse time delay as

$$
u_{m}=\tau_{m}^{-1}=\frac{1}{d s_{m} / d E}=\frac{1+a_{m}^{2}}{d a_{m} / d E} .
$$

Thus eliminating the derivative via Eq.(2.6) we obtain

$$
u_{m}=g^{2}<h_{m}\left|W W^{+}\right| h_{m}>+g^{2} \frac{<h_{m}\left|W W^{+}\right| h_{m}>}{a_{m}^{2}} .
$$

But it follows from Eq.(2.4) and the relation between $\mid f_{m}>$ and $\mid h_{m}>$ that the second term on the right hand side above is proportional to the norm $<f_{m} \mid f_{m}>$. Thus finally we get:

$$
u_{m}=g^{2}<h_{m}\left|W W^{+}\right| h_{m}>+<f_{m} \mid f_{m}>/ g^{2} .
$$

Eq.(2.9) indicates that the distribution of inverse time delays is related to the norms appearing on its rhs. Having in mind that $<h_{m}\left|W W^{+}\right| h_{m}>$ is a sum of $M$ terms:

$$
<h_{m}\left|W W^{+}\right| h_{m}>=\left|<h_{m}\right| w_{1}>\left.\right|^{2}+\ldots+\left|<h_{m}\right| w_{M}>\left.\right|^{2},
$$

where $w_{k}, k=1 . . M$, are vectors describing the coupling to the channel $k$, we can estimate the distribution of the matrix element $<h_{m}\left|W W^{+}\right| h_{m}>$ by a $\chi^{2}$ distribution with $M$ degrees 
of freedom in the GOE case and $2 M$ degrees of freedom in the GUE case respectively. On the other hand $<f_{m} \mid f_{m}>$ has a $\chi^{2}$ distribution with 1 (GOE case) or 2 (GUE) degrees of freedom. Assuming the two terms on the r.h.s of Eq.(2.9) to be independent, we obtain the distribution $P(u)$ by convolution of two $\chi_{\nu}^{2}$ distributions with different number of degrees of freedom and different means. The result can be expressed in terms of the confluent hypergeometric function ${ }_{1} F_{1}(a ; c ; x)$, sometimes called Kummer function [24]. Changing the variable to $\tau=1 / u$ we obtain the distribution of time delays

$$
P(\tau)=\frac{\exp \left[-\frac{g^{2}}{\tau}\right]_{1} F_{1}\left(\frac{M \beta}{2} ; \frac{(M+1) \beta}{2} ;\left(g^{2}-g^{-2}\right) \frac{1}{\tau}\right)}{g^{\beta(M-1)} \Gamma\left(\frac{\beta(M+1)}{2}\right) \tau^{\beta \frac{M+1}{2}+1}},
$$

where $\beta=1,2$ for orthogonal and unitary ensembles, respectively.

Figure 2 shows the mean time delay $\langle\tau\rangle$ as a function of $g$ obtained by integration of the above distribution for $\beta=2$ and $M=2,4,6$ and 16. In general mean time delay decreases with $M$. Moreover, the value of the coupling constant $g_{m}$, for which the mean delay time is maximal, decreases with the number of channels. In both limiting cases $g \rightarrow 0$ and $g \rightarrow \infty$ the mean time delay tends to zero, but the physical meaning of this fact is different. In the former case the coupling is so weak that the scattered wave is not affected by the bound system $H$. In the latter case very strong coupling causes the scattering to occur almost instantaneously.

Formula (2.11) simplifies in the special case $g=1$. Then the inverse time delays are distributed according to $\chi^{2}$ distribution with $\beta(M+1)$ degrees of freedom and the mean $\langle u\rangle=\beta(M+1) / 2$. The time delay distribution, $P(\tau)$ is then

$$
P(\tau)=\frac{1}{\Gamma\left(\frac{\beta(M+1)}{2}\right)} \tau^{-\beta \frac{M+1}{2}-1} e^{-\frac{1}{\tau}},
$$

with the mean time delay

$$
\langle\tau\rangle=2 /(\beta(M+1)-2)
$$

For large number of channels $M$ the $\chi^{2}$ distribution can be approximated by a Gaussian with the variance equal to the mean $\langle u\rangle$. Thus for $M$ large 


$$
P(\tau)=\frac{2 \alpha}{\sqrt{\pi} \tau^{2}} \exp \left\{-\alpha^{2}\left(2 / \tau-2 / \tau_{0}\right)^{2}\right\}
$$

where $\alpha^{2}=1 /(4 \beta(M+1))$ and $\tau_{0}=2 / \beta(M+1)$.

Consider now the case when the Hamiltonian $H=H(x)$ depends on some external parameter $x$ and investigate the properties of $s_{m}(x)$ keeping the energy, $E$, fixed.

Equation (2.5), putting explicitly the $x$-dependence, takes the following form

$$
\left(H(x)+\frac{g^{2}}{a_{m}(x)} W W^{+}\right)\left|h_{m}(x)>=E\right| h_{m}(x)>,
$$

and may be viewed as an eigenvalue equation for eigenvalues $E_{n}(a, x)$ of the matrix $H(x)+$ $\frac{g^{2}}{a} W W^{+}$, defined for any real parameter $a$. Then Eq.(2.15) is equivalent to a set of implicit equations

$$
E_{n}(a, x)=E ; \quad n=1,2, . . N
$$

for an unknown $a(x)$. Again due to the positivity of $W W^{+}$the equation (2.16) has at most one solution $a_{m}(x)$ for each $n$ with the total number of all possible solutions being equal to M. Moreover from Eq.(2.16) follows

$$
\frac{d a_{m}(x)}{d x}=\frac{\partial E_{m}(a, x)}{\partial x} \frac{a_{m}^{2}}{g^{2}<h_{m}\left|W W^{+}\right| h_{m}>},
$$

where $E_{m}$ denotes those eigenvalues for which (2.16) has a nontrivial solution. Combining (2.17) and (2.3) together with Eq.(2.6) yields simply

$$
v_{m}=\frac{d}{d x} s_{m}=\tau_{m} \frac{\partial E_{m}}{\partial x}
$$

providing the connection between the slope of the eigenphase with respect to external parameter $x$, the corresponding time delay and the slope of the hermitian eigenvalue problem Eq.(2.15). The generic distribution of slopes for Hermitian random matrices is Gaussian-like [17,18]. Thus the eigenphases slope distribution $P(v)$ may be obtained as a simple integral of the Gaussian and $P(\tau)$ given by Eq.(2.12) [we consider the critical coupling, $g=1$ case only]: 


$$
P(v)=\frac{1}{\sqrt{2 \pi}} \int_{0}^{\infty} P(\tau) \exp \left(-\frac{v^{2}}{2 \tau^{2}}\right) \frac{d \tau}{\tau},
$$

under the assumption that appropriate unfolding of the parameter, $x$, has been made 17, 18. The integral in (2.19) yields 25]

$$
P(v)=\frac{\beta(M+1)}{\sqrt{8 \pi} v^{\beta(M+1) / 2+1}} \mathcal{D}_{-\beta(M+1) / 2-1}(1 / v),
$$

where $\mathcal{D}_{p}(z)$ is a shorthand notation for the product of the parabolic cylinder function, $D_{p}(z)$, 25 with the exponential

$$
\mathcal{D}_{p}(z)=\exp \left(z^{2} / 4\right) D_{p}(z)
$$

Using the series representation of $\mathcal{D}_{p}(z)$ for small $z$ one easily verifies that the large $|v|$ tail of $P(v)$ decays algebraically as $P(v) \propto 1 /|v|^{\beta(M+1) / 2+1}$. The asymptotic form of $\mathcal{D}_{p}(z)=$ $z^{p}\left[1+O\left(z^{-2}\right)\right]$ [25] valid for large $z$ yields regular behaviour of $P(v)$ for $|v|$ small.

Let us recall that the distribution of level slopes with respect to an external parameter, $x$, for a bounded generic system is Gaussian [17, 18]. It is remarkable that the corresponding slope distibution for the eigenphases of the $S$ matrix reveals the algebraic tails. As we shall demonstrate in the numerical example below, for large $M$ the algebraic tail asymptotic form appears for very large $|v|$ only, while the center of the distribution resembles a Gaussian. One may verify, using the asymptotic form pf $P(\tau)$ given by Eq.(2.14) that the Gaussian distribution for $P(v)$ is recovered in the $M \rightarrow \infty$ limit.

A similar analysis can be carried out also for curvature distribution. In the limit of large $M$ it can be shown in such a way that the curvature distribution tends to the generalized Cauchy one, as for the bounded systems [18].

\section{NUMERICAL RESULTS - HAMILTONIAN APPROACH}

We discuss the numerical results obtained for the slopes with respect to the energy, $E$, first. As discussed above they are directly related to possible time delays in the scattering process and are, therefore, of particular interest. The time delay distributions, $P(\tau)$, 
obtained numerically by diagonalizing the $S$ (1.2) for various number of channels $M$ and $g=1$ in the GUE case are presented in Fig. 3 and compared with the formula (2.12). The dimension of the internal Hamiltonian was taken $N=100$, energy $E$ was set to zero and the data where obtained from 15000 generated $S$ matrices. Fine agreement of the numerical results with the formula (2.12) does not depend on the energy and the number of eigenstates, provided $N>>M$ and $E$ is not too large. Figure (雨) shows a comparison of the distribution $P(\tau)$ obtained for $g \neq 1$ and the formula (2.11). Again the agreement is quite satisfactory.

Analogous results for the GOE case and $g=1$ are plotted on the Fig. (dashed line). The agreement with the prediction (2.12) is not so nice as in the GUE case. Especially for small $M$ a discrepancy is significant. Importantly, it may be removed when we use the formula (2.12) with one additional degree of freedom, i.e.:

$$
\tilde{P}(\tau)=\frac{1}{\Gamma\left(\frac{M+2}{2}\right)} \tau^{-\frac{M}{2}-2} e^{-\frac{1}{\tau}} .
$$

The corresponding average time delay is given by Eq.(2.13) with $\beta=1$ and $M \rightarrow M+1$ substitutions. We are not able to give a plausible explanation for this additive degree of freedom. The numerical results demonstrate clearly, however, that it is superior to Eq.(2.12) and leads to an excelent agreement with the numerical data - see Fig.5.

The formula (3.1) implies that the tail of the distribution for GOE behaves as $\tau^{-M / 2-2}$. In particular, for $M=3$ we obtain for the time delay distribution $P(\tau) \approx \tau^{-7 / 2}$, in a full agreement with the experimental finding in 26].

Similar numerical tests may be also performed for the velocity distribution in the case of a parametric dependence in order to test the formulae (2.19), (2.20). To this end we have to introduce a parametric dependence into the basic model of Eq.(1.2-1.3). One can, in principle, discuss several possible cases, the parameter $x$ may affect either the bound system only [described by $H_{k l}$ in Eq.(1.3)] or the decay part, i.e. the bound-continuum coupling $W$, or both. In a generic case, arguably, both parts of the effective Hamiltonian will be affected. We consider the case when the internal dynamics only is $x$ dependent, i.e., $H=H(x)$. This situation corresponds directly to predictions obtained in the previous section. A similar 
approach has been adopted in the treatment of time reversal symmetry breaking influence on the conductance fluctuations [21].

The parametric $x$-dependence is taken in a generic form: $H=H_{1} \cos (x)+H_{2} \sin (x)$ [18] where $H_{i}$ are drawn independently from the same ensemble of random matrices. The trigonometric form assures that the mean density of states remains the same for all $x$ and the motion of bound levels as a function of $x$ is stationary [18]. The slopes have been calculated by a finite difference in $x$ from eigenphases of $S$. As before more than $15000 S$-matrices were used for the averaging.

In the GUE case the agreement between the numerical data and the analytic prediction for the slope distribution, Eq.(2.20) is again remarkable - see Fig. 6. Note strongly nonGaussian character of the obtained distribution. In the semi-logarithmic scale used in Fig. 6 a Gaussian would take an inverted parabola shape. The distributions show an algebraic tail $P(v) \propto v^{-(M+2)}$ for small $M$. For $M$ large a center of the distribution resembles a parabola (i.e., a Gaussian distribution in the linear scale) followed by a straight line in the semilogarithmic plot indicating a regime of exponential behaviour, $P(v) \propto \exp (-\gamma|v|)$. The numerical data are insufficient to detect a transition to a possible algebraic tail for $|v|$ large. For $M$ even larger (not shown) the regime of Gaussian behaviour broadens, the exponential behaviour moves to the tails indicating the transiton to the semiclassical limit.

Consider now GOE internal dynamics. Similarly as for the time delays - the agreement with the numerical data is admirable if the distribution (2.19) is evaluated with the "adhoc" improved $\tilde{P}(\tau)$ given by Eq. (3.1) - compare Fig. 17. As before the "proper" $P(\tau)$ [Eq.(2.12)] with $\beta=1$ used in arriving at Eq.(2.20) leads to a theoretical prediction with clear disagreement with the numerical data for small $M$ (not shown). The corrected expression is obtained from Eq.(2.20) by $\beta=1$ and $M \rightarrow M+1$ substitutions. The distributions obtained are similar in shape to those corresponding to GUE case, with the algebraic tail of the form $P(v) \propto v^{-M / 2-2}$ seen clearly for $M$ small. For the largest number of channels plotted $M=16$ we again observe the Gaussian center with exponential tails in similarity with the GUE distribution. 
To complete the discussion of the parametric dependence in the Hamiltonian based approach let us pass now to the curvature distributions. Numerical tests performed for the curvatures $K=d^{2} s / d E^{2}$ are presented in Fig.8. Note the quite good agreement of the numerical data with the generalized Cauchy distribution [18]. The double-logarithmic scale used enhances the small and large $|K|$ behaviour. For large $|K|$ the agreement is excellent confirming the universality of the large curvature tail behaviour also for the present scattering system. On the other hand one observes a slight excess of small curvatures (and the corresponding lack of "medium" curvatures). It is a clear indication that $M=16$ is not sufficient to realize fully the semiclassical limit. Using the analogy with the bound system level dynamics [18] one may conclude that avoided crossings between eigenphases (as a function of the energy, E) are still partially isolated.

The curvature distribution with respect to the external parameter is plotted in Fig.9. Comparing to the previous case, a better agreement with the generalized Cauchy distribution is observed indicating that the semiclassical regime is reached faster when the motion of eigenphases as a function of $x$ is considered. A similar qualitative conclusion may be reached considering the distribution of time delays and of the slopes with respect to the parameter $x$.

For $M$ smaller one observes stronger deviations from the universal Cauchy distribution (not shown). The explicit dependence on the number of open channels in that case has obviously the same origin as the corresponding dependence in the case of slope (or time delay) distributions.

\section{RANDOM PARAMETRIC S-MATRIX}

As mentioned in the introduction one often employs random ensembles to directly model properties of $S$ matrices [10, 12, 13]. One may envision a similar approach for the study of the parametric statistics. To this end one has to define the parametric dependence of $S$ matrices directly, without reference to the underlying bounded dynamics. Obviously, there 
is some ambiguity here, there are several possible choices. The ideal approach should be conceptually simple and, at the same time, lead to the same distributions as those obtained for the Hamiltonian based approach. After all in both cases the parameter independent matrices belong to generalized circular ensembles [9]. We shall see below that the simplest possibilities agree with the Hamiltonian approach in the limit of large number of channels (semiclassical limit) only.

The first approach proposed takes $S$ matrices in the form:

$$
S(x)=S_{0} \exp (i x V)
$$

where $S_{0}$ is drawn from the appropriate circular ensemble (COE or CUE) of a given rank $M$ while $V$ is a Hermitian matrix (rank $M$ ), independent of $S_{0}$ and drawn from GOE or GUE, respectively. Then the eigenphases at $x=0$ are the phase shifts of $S_{0}$. The slopes and curvatures of $S$ are easily defined as the corresponding derivatives at $x=0$.

Such a form of a parametric dependence is "borrowed" from the typical form of a Floquet operator corresponding to quantum maps (e.g., for a famous kicked rotator or for the kicked top - for a detailed introduction to such problems see [27]). While in [28] the form of the Floquet operator was determined by the dynamics of the system, here we assume a random $S_{0}$, as discussed above.

Eq. (4.1) implies a Gaussian distribution of slopes, since the slopes of eigenphases of $S$ at $x=0$ are given by diagonal elements of matrix $V$ represented in the eigenbasis of $S_{0}$. The latter are Gaussian distributed as we have taken $S_{0}$ and $V$ independently. The Gaussian character of the velocity distribution for the model (4.1) is independent on the matrix size $M$ and holds for both COE and CUE. Recalling the results obtained in the Hamiltonian model we see that the statistical predictions for eigenphase slope distribution coincide only in the $M \rightarrow \infty$ limit.

The random $S$ matrices belonging to a given ensemble of random unitary matrices $(\mathrm{COE}$, CUE) may be generated (for arbitrary $M$ ) by drawing the generalized Euler angles from the appropriate probability distribution [29]. Similarly one may construct the way to introduce 
the parametric dependence by considering the infinitesimal changes of the generalized Euler angles [30]. Our numerical results indicate that if one assumes that all $M^{2}-1$ angles are affected by the perturbation in a same way, the distribution of slopes is Gaussian for arbitrary $M$.

Results received of $10^{4}$ random unitary COE matrices with $M=40$ are presented in Fig. 10. Gaussian character of the "velocity" distribution, obtained in this case, is not at all typical of any parametric dynamics defined for random unitary matrices. On contrary, one may construct many kinds of parametric dynamics in the space of unitary matrices leading to non-Gaussian distributions $P(v)$. Exemplary data, represented in the figure by triangles, where obtained for a model in which only $M$ diagonal Euler angles have beed varied. For small $M$ the distributions obtained are closer to the results of the Hamiltonian model (1.2) than the pure Gaussian distribution. Similar results hold also for CUE case.

Let us consider now the second derivatives of eigenphases with respect to $x$. For a Floquet operator corresponding to the kicked top model, the parametric curvature distribution has been studied already [28] and shown [18] to obey the generalized Cauchy distribution with quite a good accuracy for all three universality classes. The numerical simulations performed by us indicate that for $M$ large (semiclassical limit) the same property holds for the $S$ matrix models described above.

The simple propositions discussed above failed to reproduce the distribution of slopes with respect to the external parameter, $x$, obtained for small $M$ within the Hamiltonian $S$-matrix model. However this goal may be simply obtained by a slight modification of assumption concerning the matrix $V$ in Eq.(4.1). As discussed above the distribution of slopes of $S$ matrix eigenphases is equivalent to the distribution of diagonal elements of $V$ in the eigenbasis of $S_{0}$. Instead of choosing $V$ to belong to the appropriate for the given symmetry Gaussian random ensemble one may chose $V$ from the ensemble defined by the desired distribution of diagonal elements, Eq.(2.19). This defines the distribution of $M$ elements of $V$ leaving the remaining $M(M-1) / 2$ elements undefined (in a chosen basis). This additional freedom may be utilized to make this ensemble less artificial than it seems 
at first. While this aposteriori procedure may be hardly described as an elegant one, it gives an indication of the properties of the required ensemble of $V$ matrices. In particular, following the discussion presented in Section II, it is clear that the construction of such an ensemble may involve scalar products of unnormalized vectors in $M$-dimensional space [compare Eq. (2.10)]. Hopefully one may define the random ensemble for $S$ matrices of the form (4.1) which is universal, i.e., reproduces not only the distribution of slopes but also of all other statistical measures involving the parameter $x$. This would allow to use the "direct" $S$ matrix approach instead of the Hamiltonian one, also for the problems involving the external parametric dependence.

\section{CONCLUDING REMARKS}

In this work we have considered the statistical properties of derivatives of the $S$ matrix eigenphases with respect to the energy, $E$, as well as with respect to some external parameter $x$. Within the Hamiltonian approach we were able to derive, using simple heuristic arguments, the analytic distributions for the time delay valid for arbitrary number of open channels $M$ and arbitrary value of the coupling constant $g$. An analytic distribution of slopes with respect to an external parameter has been also obtained. A comparison with the numerical simulations have shown good agreement of the theory with the numerical data in the CUE case. The discrepancies observed for the COE case may be removed by an "ad-hoc" modification of the proposed expression.

When the main part of this work has been finished we have been informed that in the CUE case the same formulae for the time delay distribution have been obtained by Fyodorov and Sommers using supersymmetry calculus [31]. We hope that the supersymmetric approach will be able in the future to verify the proposed distributions also for the time-reversal invariant (COE) model.

We have verified numerically that the curvature distribution (i.e., the distribution of second derivatives of eigenphases with respect to energy) obeys the generalized Cauchy 
distribution [18] in the semiclassical limit ( $M$ large).

Construction of the parametric dependence directly for the random $S$ matrices belonging to a given random ensemble has been shown to be ambiguous. The natural choices for the dynamics lead to Gaussian velocity distribution for arbitrary number of open channels. Then the agreement with the Hamiltonian based approach is obtained in $M \rightarrow \infty$ limit only.

J.Z. thanks D. Delande for discussions. We acknowledge interesting exchanges with Y. Fyodorov and are grateful for informing us of his results prior to the publication.

Laboratoire Kastler Brossel, de l'Ecole Normale Superieure et de l'Universite Pierre et Marie Curie, is unité associée 18 du CNRS. This work was supported by the Polish

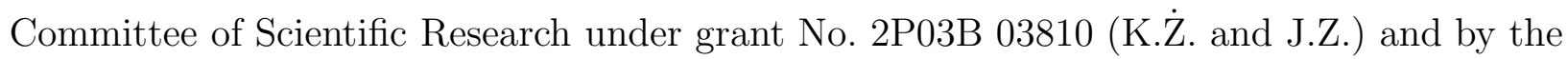
GA AS No. 148409 (P.Š.). 


\section{REFERENCES}

[1] U. Smilansky in Chaos and quantum physics, edited by M.-J. Giannoni, A. Voros and J. Zinn-Justin, Les Houches Summer School, Session LII (North-Holland, Amsterdam, 1991).

[2] C. H. Lewenkopf and H. A. Weidenmüller, Ann. Phys. (N.Y.) 212, 53 (1991).

[3] C. Mahaux and H. A. Weidenmüller, "Shell-Model Approach to Nuclear Reactions", North-Holland, New York, (1969).

[4] J. J. M. Verbaarschot, H. A. Weidenmüller, and M. R. Zirnbauer, Phys. Rep. 129, 367 (1985).

[5] C.M. Marcus, A.J. Rimberg, R.M. Westervelt, P.F. Hopkins, A.C.Gossard, Phys. Rev.Lett, 69, 506, 1992.

[6] A.M. Chang, H.U. Barnager, L.N. Pfeiffer, K.W. West, Phys. Rev. Lett. 73, 2111 (1994).

[7] O. Bohigas in Chaos and quantum physics, edited by M.-J. Giannoni, A. Voros and J. Zinn-Justin, Les Houches Summer School, Session LII (North-Holland, Amsterdam, 1991).

[8] M. L. Mehta, Random Matrices, II ed. (Academic, New York, 1991).

[9] P. W. Brouwer, Phys. Rev. B51, 16878 (1995).

[10] P. A. Mello, P. Pereyra, and T. H. Seligman, Ann. Phys. (N.Y.) 161, 254 (1985).

[11] F. J. Dyson, J.Math, Phys. 3, 140 (1962).

[12] Y. Imry, Europhys. Lett. 1249 (1986).

[13] K. A. Muttalib, J.-L. Pichard, and A. D. Stone, Phys. Rev. Lett. 59, 2475 (1987).

[14] B. L. Altschuler and B. Shklovskii, Zh. Eksp. Teor Fiz. 91, 220 (1986) [ Sov. Phys. JETP 64, 127 (1986)]; P. A. Lee, D. Stone, and H. Fukuyama, Phys. Rev. B35, 1039 
(1987); for a review see e.g., Mesoscopic Phenomena in Solids, B. L. Altshuler, P. A. Lee and R. A. Webb eds., (North-Holland 1991). York, 1991.

[15] R. A. Jalabert, J-L. Pichard, J.Phys. I France, 5, 287, (1995).

[16] P. Gaspard, S. A. Rice and K. Nakamura, Phys. Rev. Lett. 63, 930 (1989); P. Gaspard, S. A. Rice H. J. Mikeska and K. Nakamura, Phys. Rev. A42, 4015 (1990).

[17] B. D. Simons and B. L. Altshuler, Phys. Rev. Lett. 70, 4063 (1993); Phys. Rev. B48, $5422(1993)$.

[18] J. Zakrzewski and D. Delande, Phys. Rev. E 47, 1650 (1993).

[19] F. von Oppen, Phys. Rev. Lett.73, 798 (1994).

[20] A.M.S. Macedo, Phys. Rev. E 50, R659 (1994).

[21] Z. Pluhař, H. A. Weidenmüller, J. A. Zuk, C. H. Lewenkopf, Phys. Rev.Lett, 732115 (1994).

[22] E. Doron, U. Smilanski, A. Frenkel, Physica D 50, 367 (1991).

[23] E. Doron and U. Smilansky, Nucl. Phys. A545, 455c (1992).

[24] J. Spanier and K.B. Oldham An Atlas of Functions, (Hemisphere Publishing Corp., Washington 1987).

[25] I. S. Gradshteyn and I. M. Ryzhik, Tables of Integrals, Series and Products, (Academic, New York, 1980).

[26] H. Alt at al: Phys. Rev. Lett. 74, 62 (1995).

[27] F. Haake, Quantum Signatures of Chaos (Springer, Berlin 1991).

[28] D. Saher, F. Haake and P. Gaspard, Phys. Rev. A44, 7841 (1991).

[29] K.Życzkowski, M.Kuś, J.Phys. A 27, 4235 (1994). 
[30] K.Życzkowski, M.Kuś, Phys. Rev. E 53, 319 (1996).

[31] Y. Fyodorov and H. J. Sommers, unpublished. 


\section{FIGURES}

FIG. 1. Scheme of the scattering system. A system containing $N$ bounded states and described by a Hamiltonian $H$ is coupled via matrices $W$ and $W^{\dagger}$ to two waveguides with $M_{1}$ and $M_{2}$ open channels. The scattering in the system is thus characterized by a $M \times M$ matrix $S$, where $M=M_{1}+M_{2}$.

FIG. 2. Dependence of the mean time delay $\langle\tau\rangle$ on the coupling constant $g$ for $\beta=2$. Number of channels $M$ labels each curve.

FIG. 3. Time delay distributions, $P(\tau)$, for GUE internal dynamics, $g=1$ and for different number of open channels, $M$ as indicated in the Figure. The numerical data (histograms) and the theoretical distributions (solid lines), $P_{2}(\tau)$, Eq.(2.12) are presented as a function of $\tau /\langle\tau\rangle$ to facilitate comparison of distribution shape for different $M$. According to Eq. $2.13<\tau>$ is inversely proportional to $M$.

FIG. 4. Time delay distributions, $P(\tau)$, for GUE internal dynamics and g.ne.1: a) $g=0.5, M=4$; b) $g=2.0, M=6$. Solid line represents the theoretical prediction as given by Eq.(2.11).

FIG. 5. Time delay distribution, $P(\tau)$, for time reversal invariant (GOE) internal dynamics, $g=1$ and for different number of open channels, $M$ as indicated in the Figure. The numerical data (histograms) are compared with both the analytical prediction, Eq.(2.12), (dashed lines) and the corrected prediction, Eq.(3.1) (represented by a solid line).

FIG. 6. Numerical data for distribution of slopes (with respect to external parameter) for GUE internal dynamic and $g=1$ plotted in the semilogarithmic scale. The theoretical prediction, Eq. (2.20) is shown as a full line and the number of channels is indicated in each graph. Note strongly non-Gaussian shape of the distribution.

FIG. 7. Same as in the previous figure but for GOE internal dynamic. The theoretical prediction is obtained by the modification of Eq. (2.20) discussed in the text. 
FIG. 8. Distribution of second derivatives of eigenphases of $S$ matrix with respect to energy, $K=d^{2} s / d E^{2}$ obtained numerically for the case of $M=16$ open channels, (a) for GUE internal dynamics, (b) for GOE dynamics. The solid (dashed) lines in both panels represent the generalized Cauchy distributions corresponding to the GOE (GUE) case respectively.

FIG. 9. As in the previous figure for the curvature with respect to an external parameter, $K=d^{2} s / d x^{2}$.

FIG. 10. Distribution of slopes of eigenphases with respect to the perturbation parameter $x$ of random unitary matrices of size $M=40$ typical of $\mathrm{COE}$; a) parameter $x$ controls variations of all $M^{2}-1$ Euler angles $(\triangle)$; b) only $M$ diagonal angles are varied $(\diamond)$. 


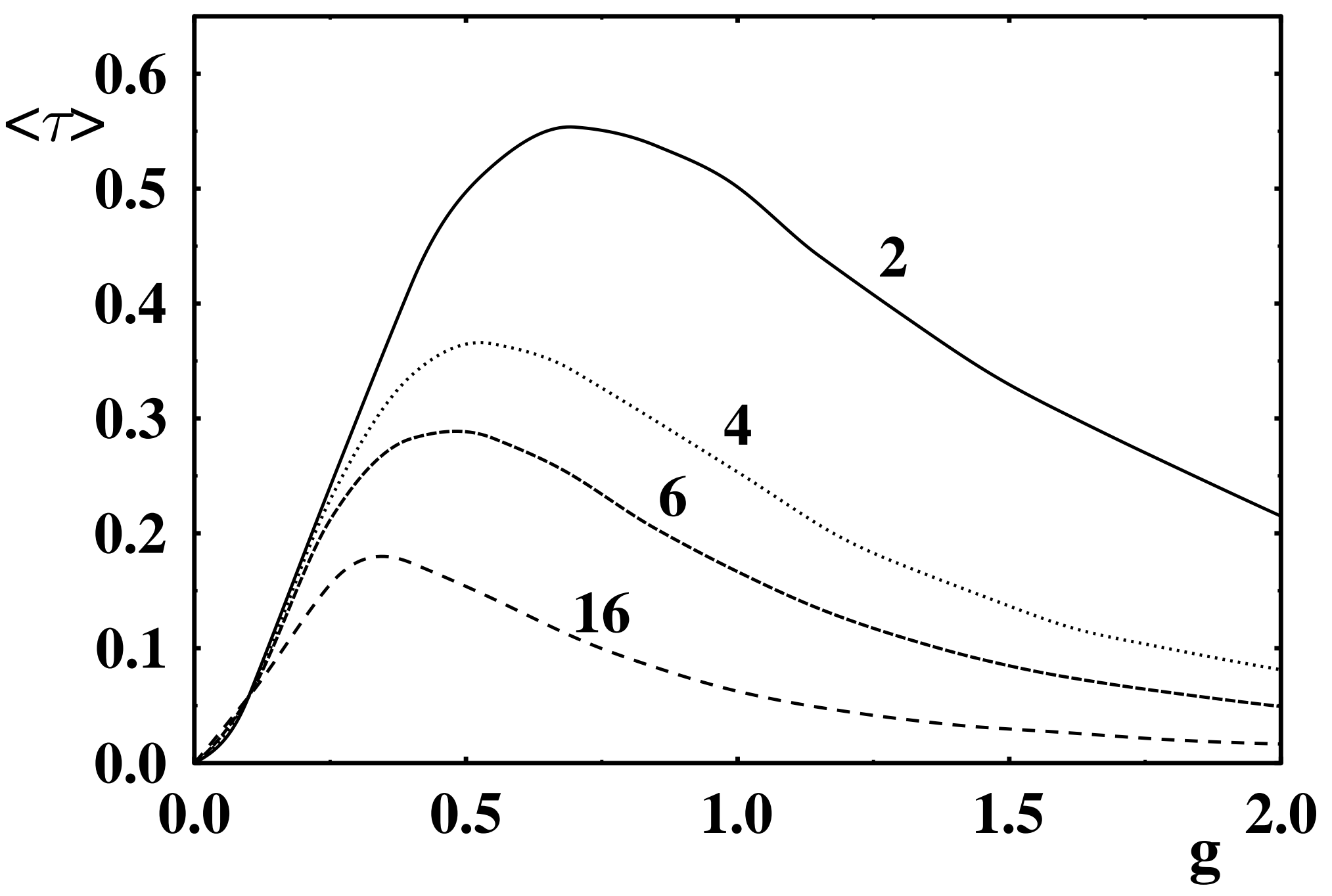




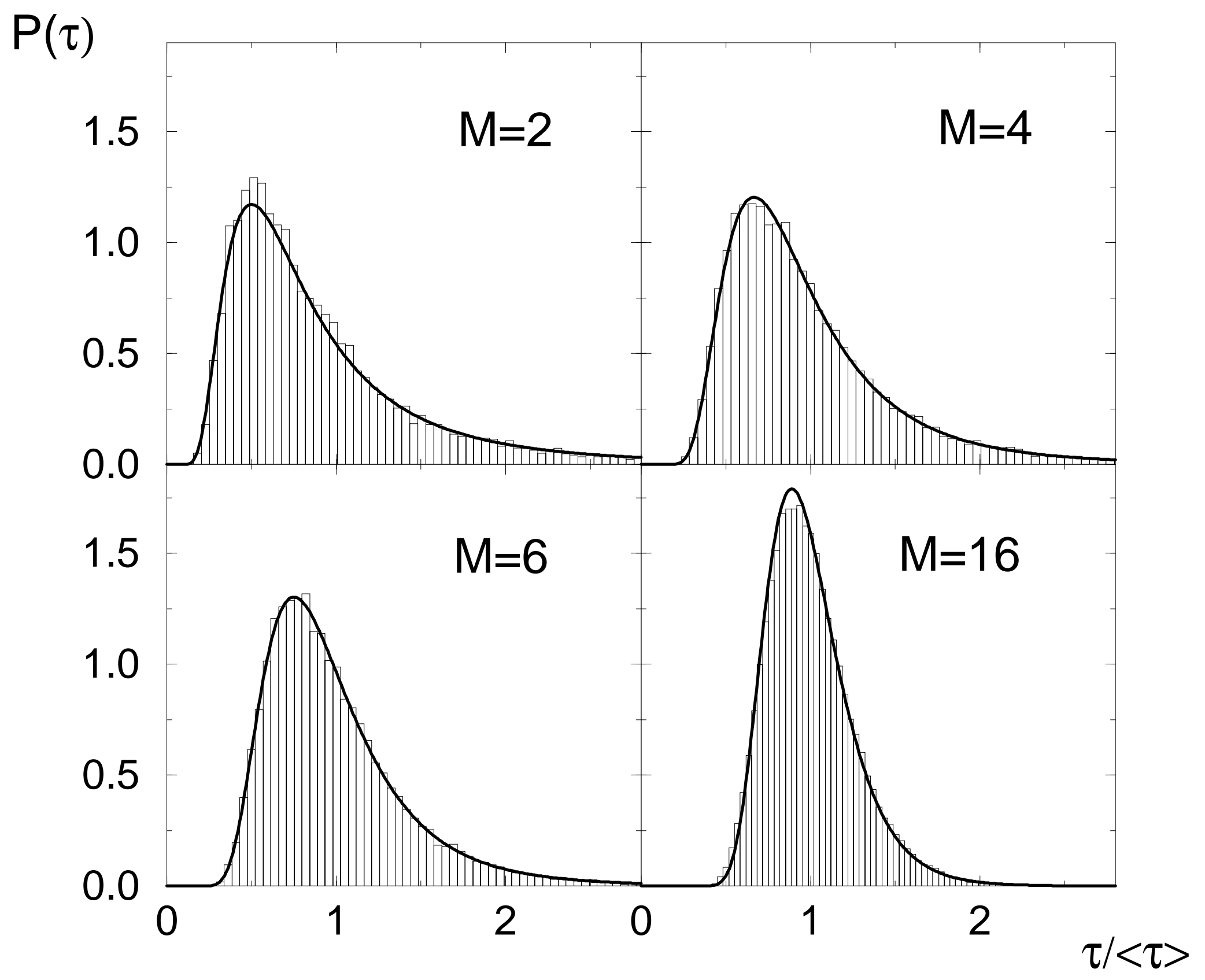




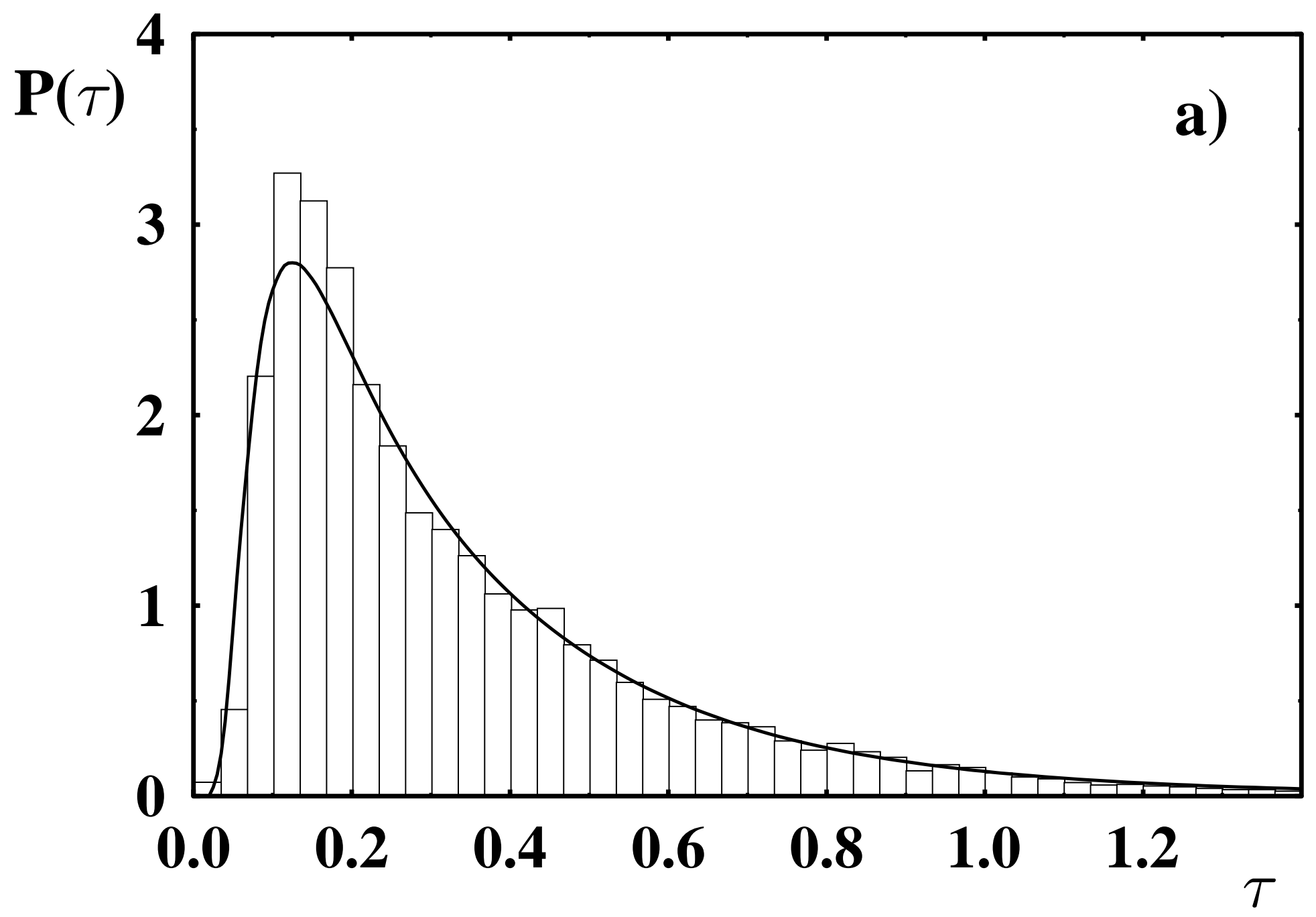




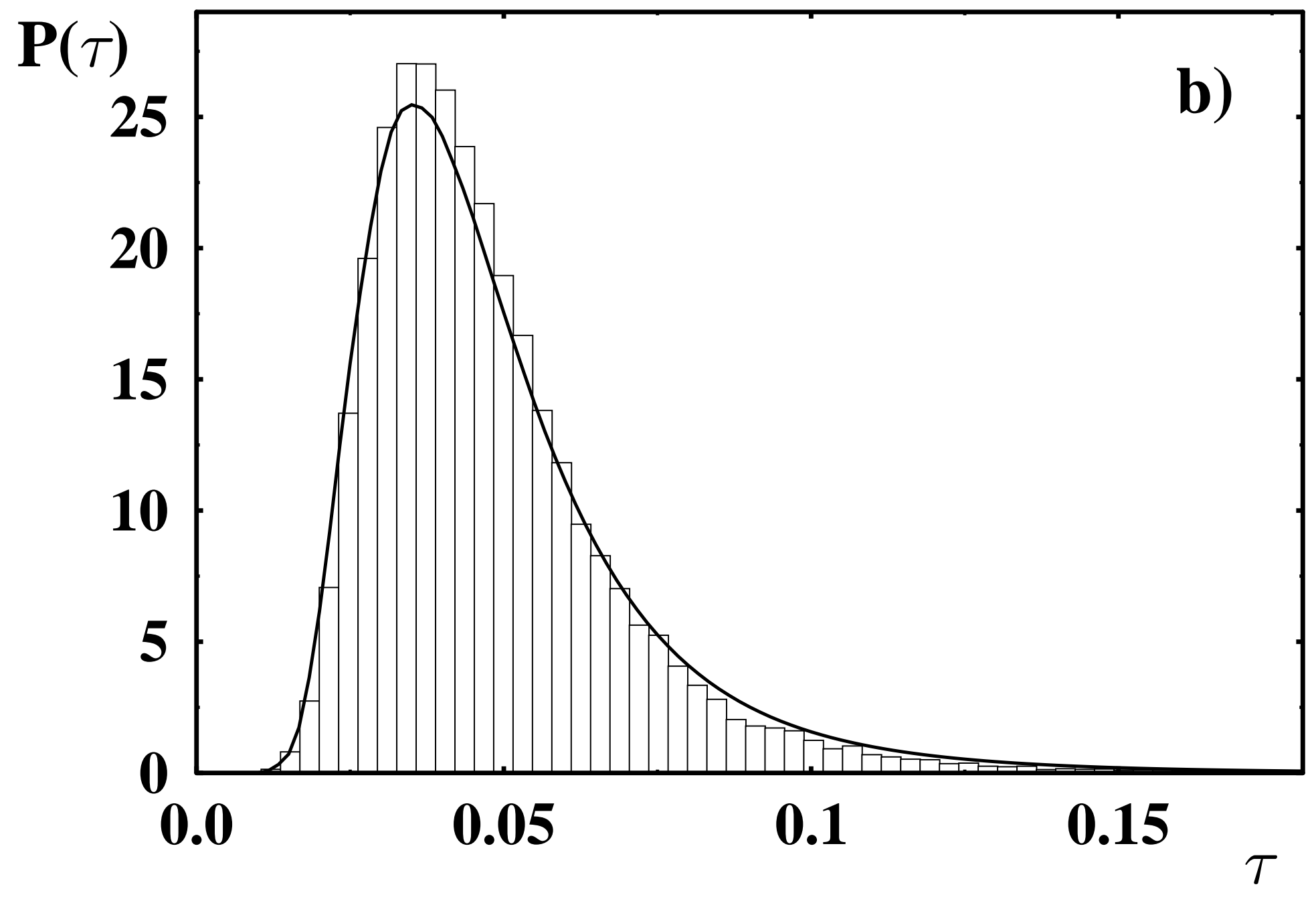




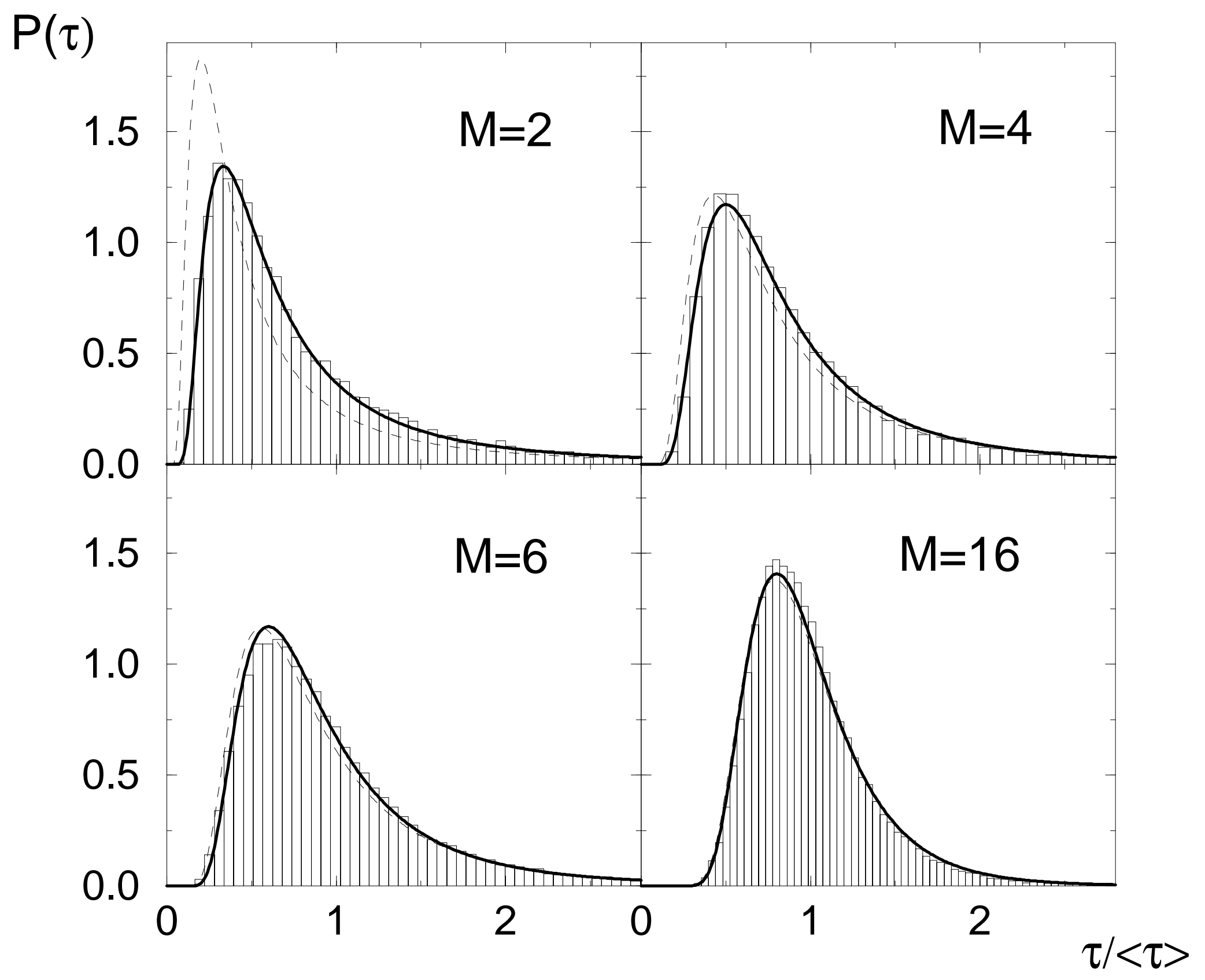



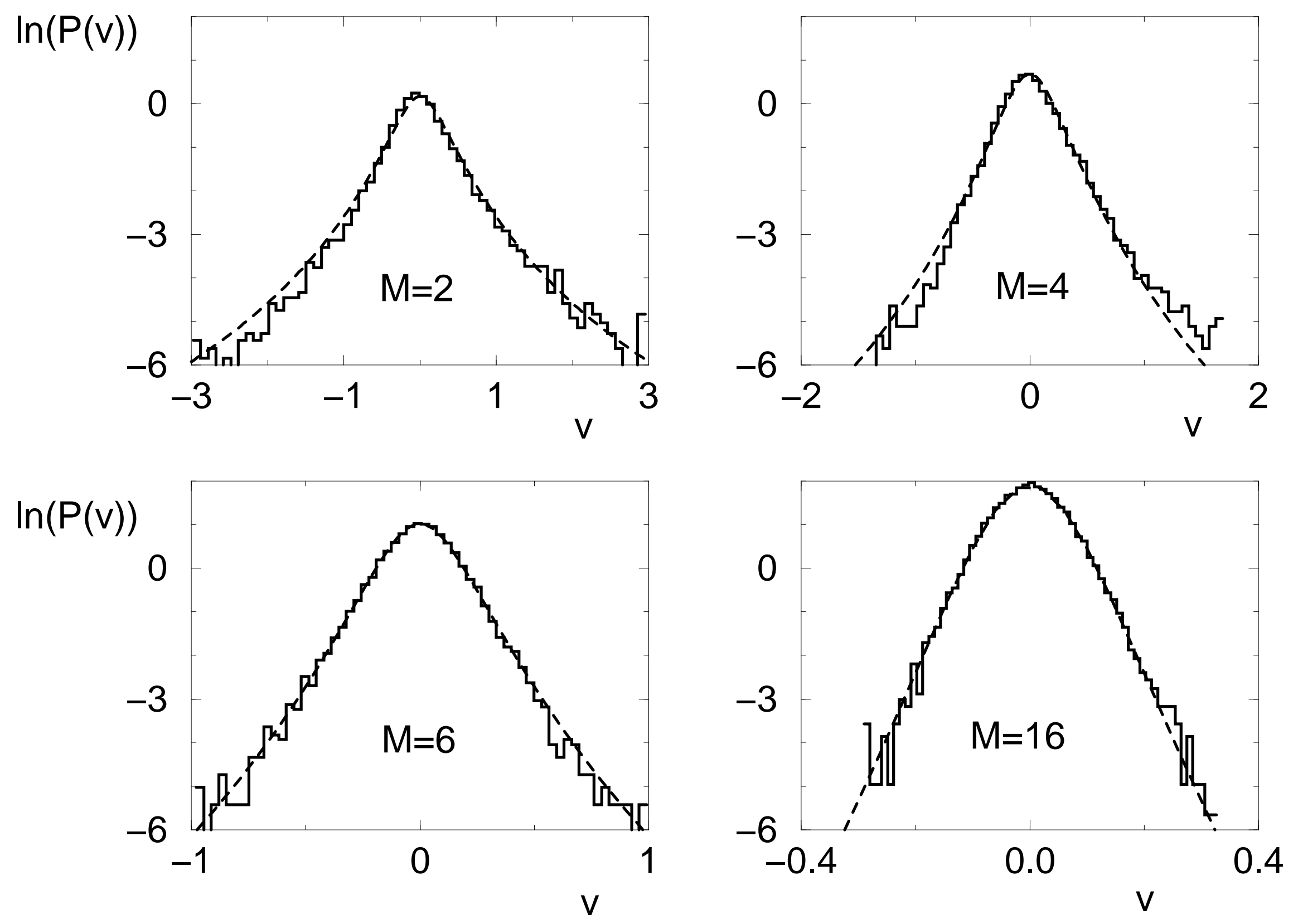

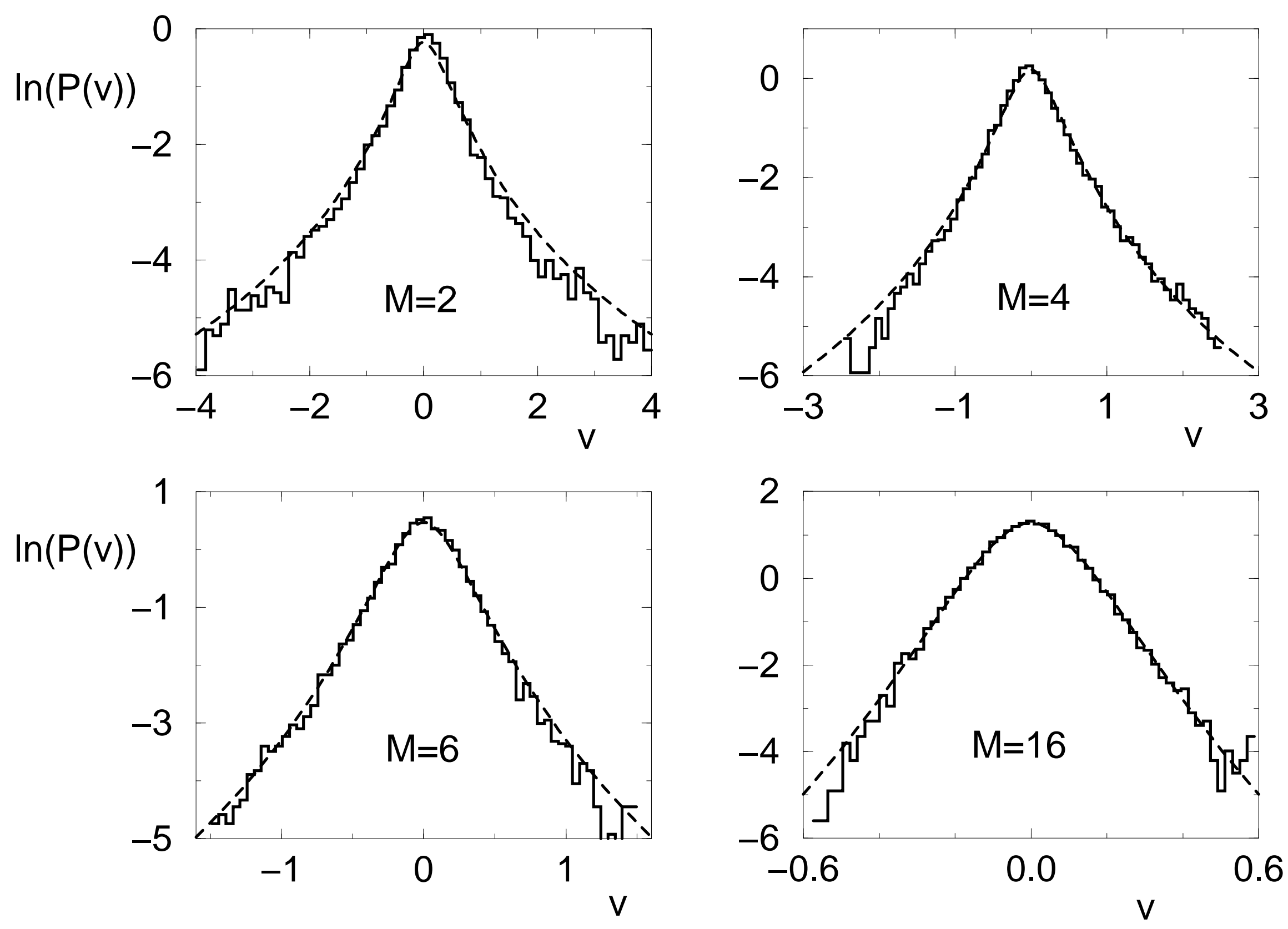


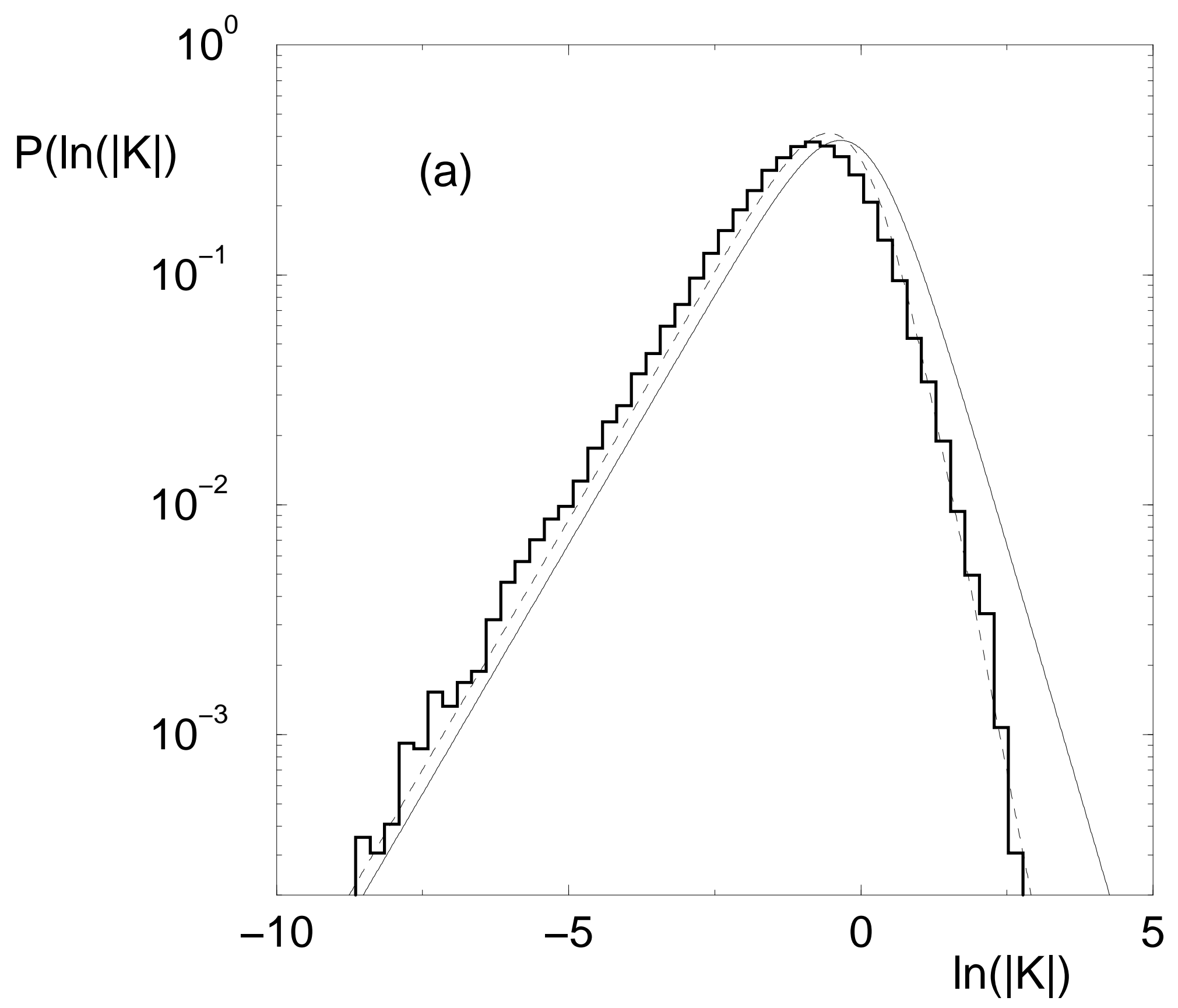




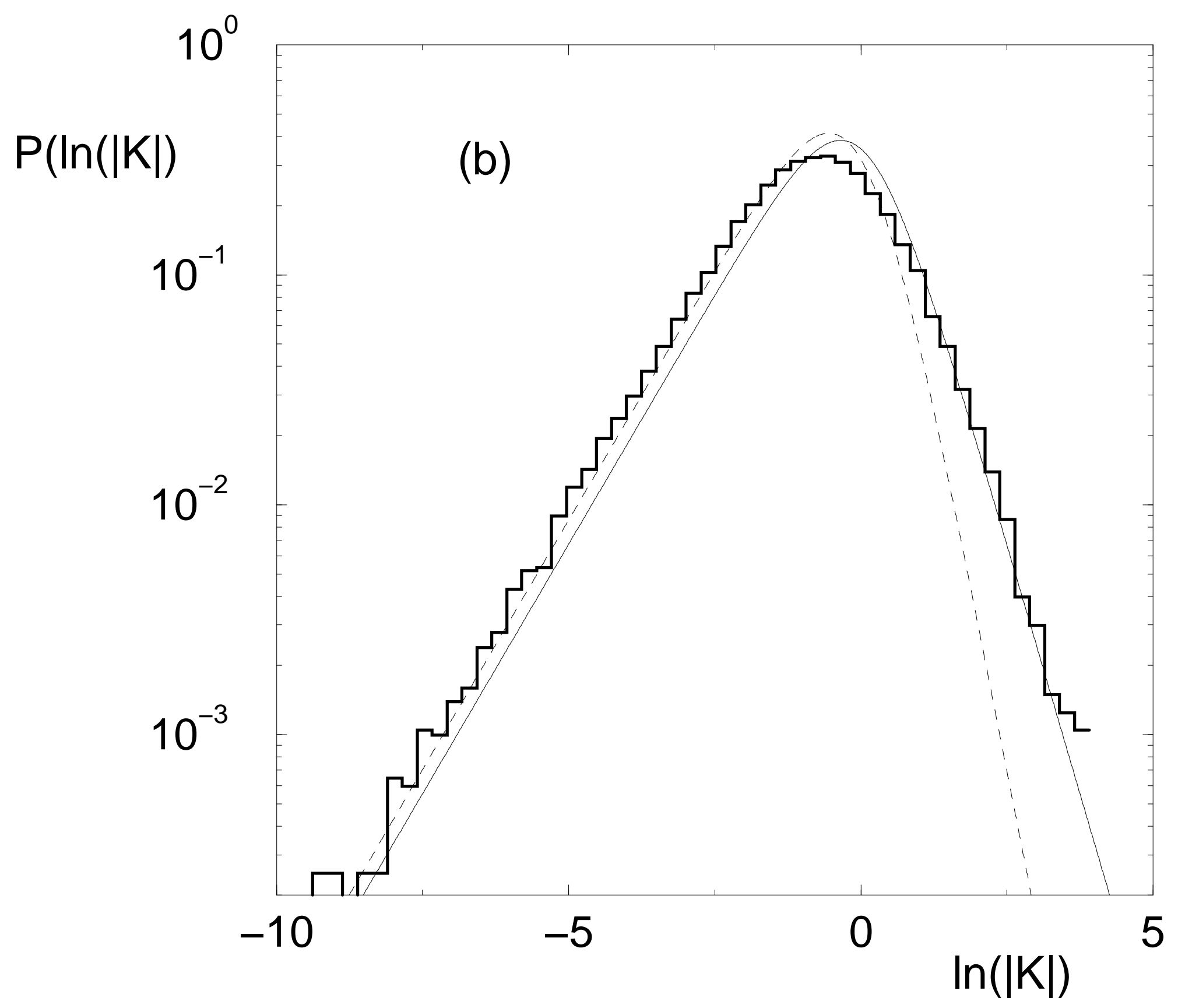




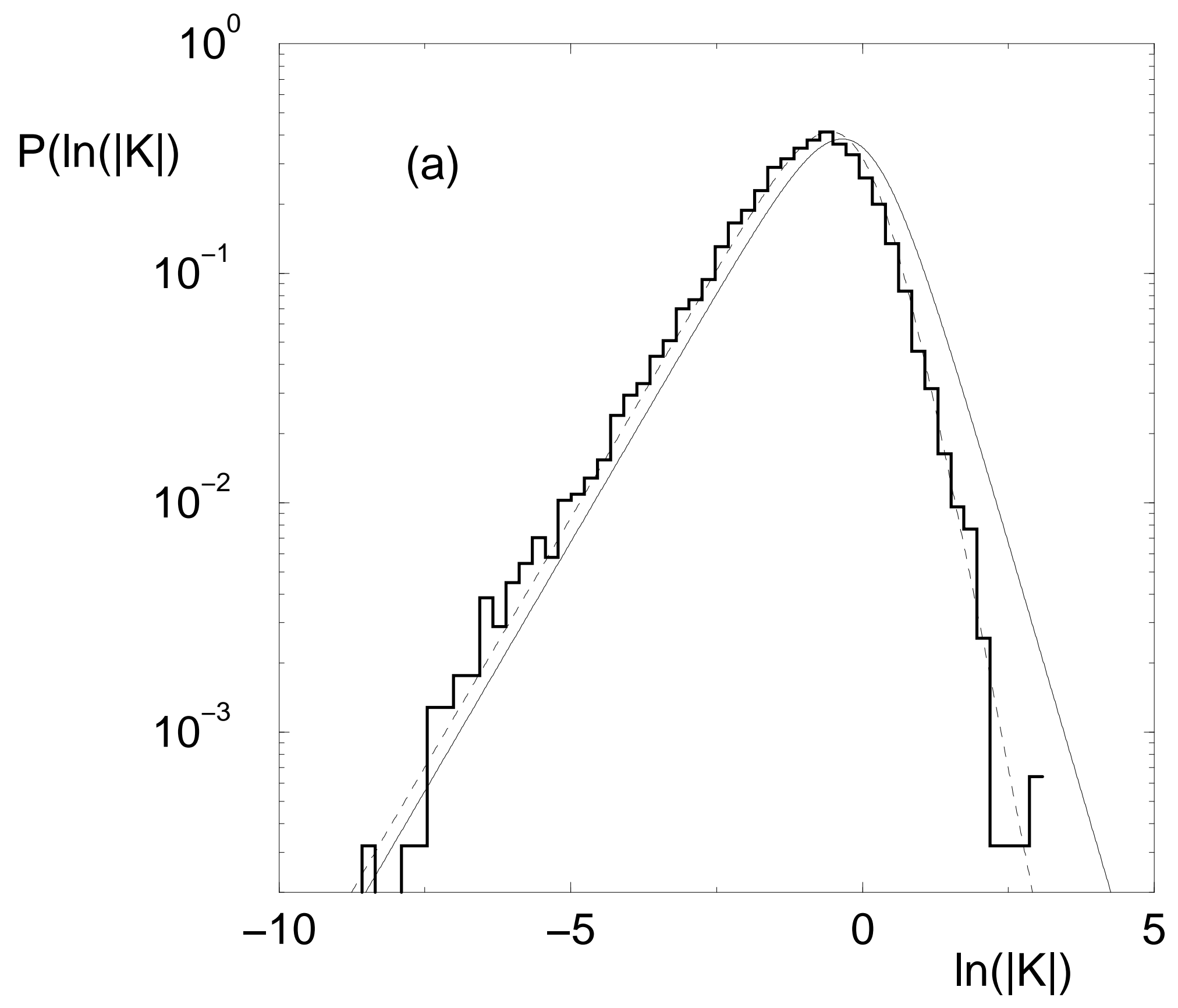




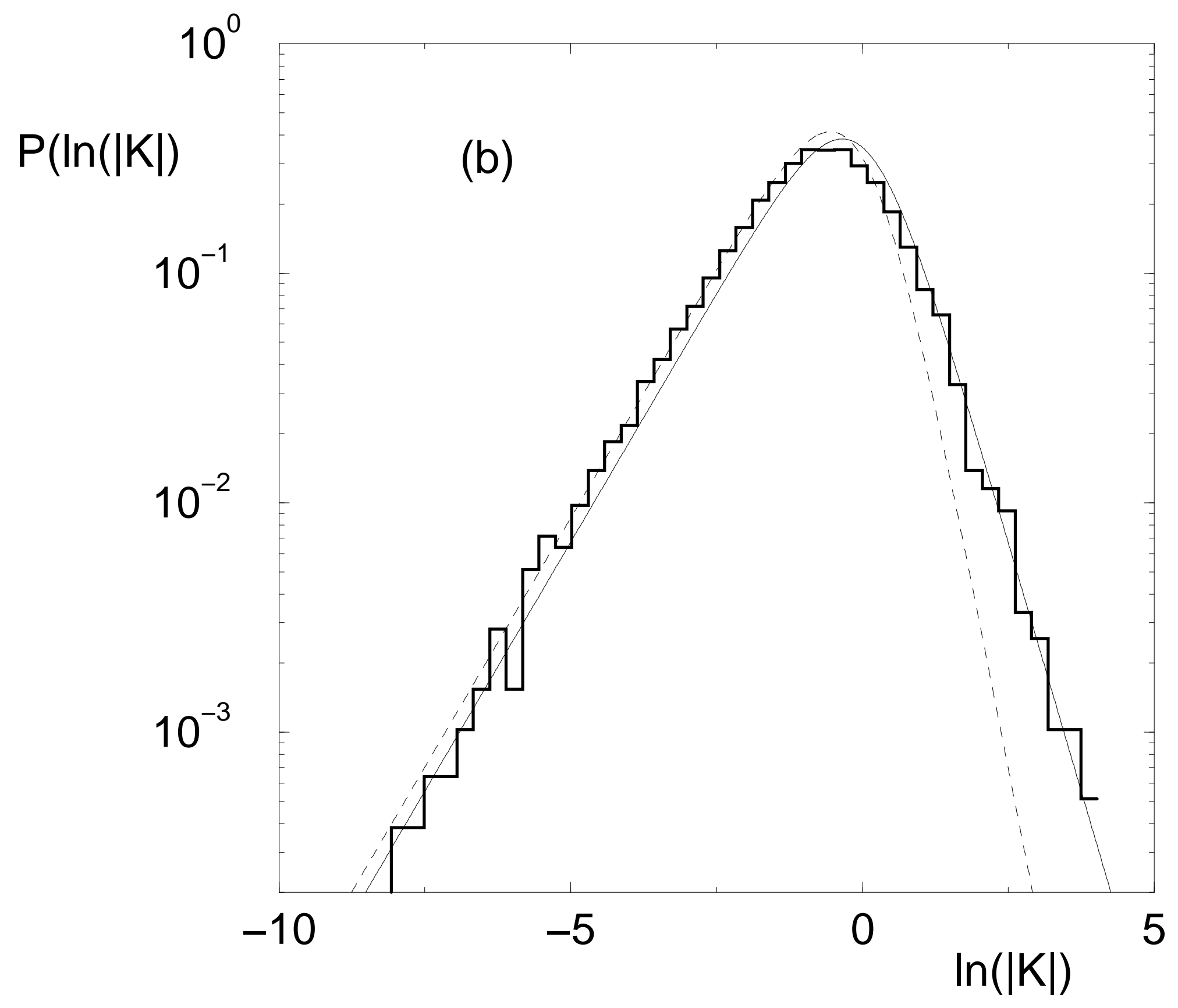




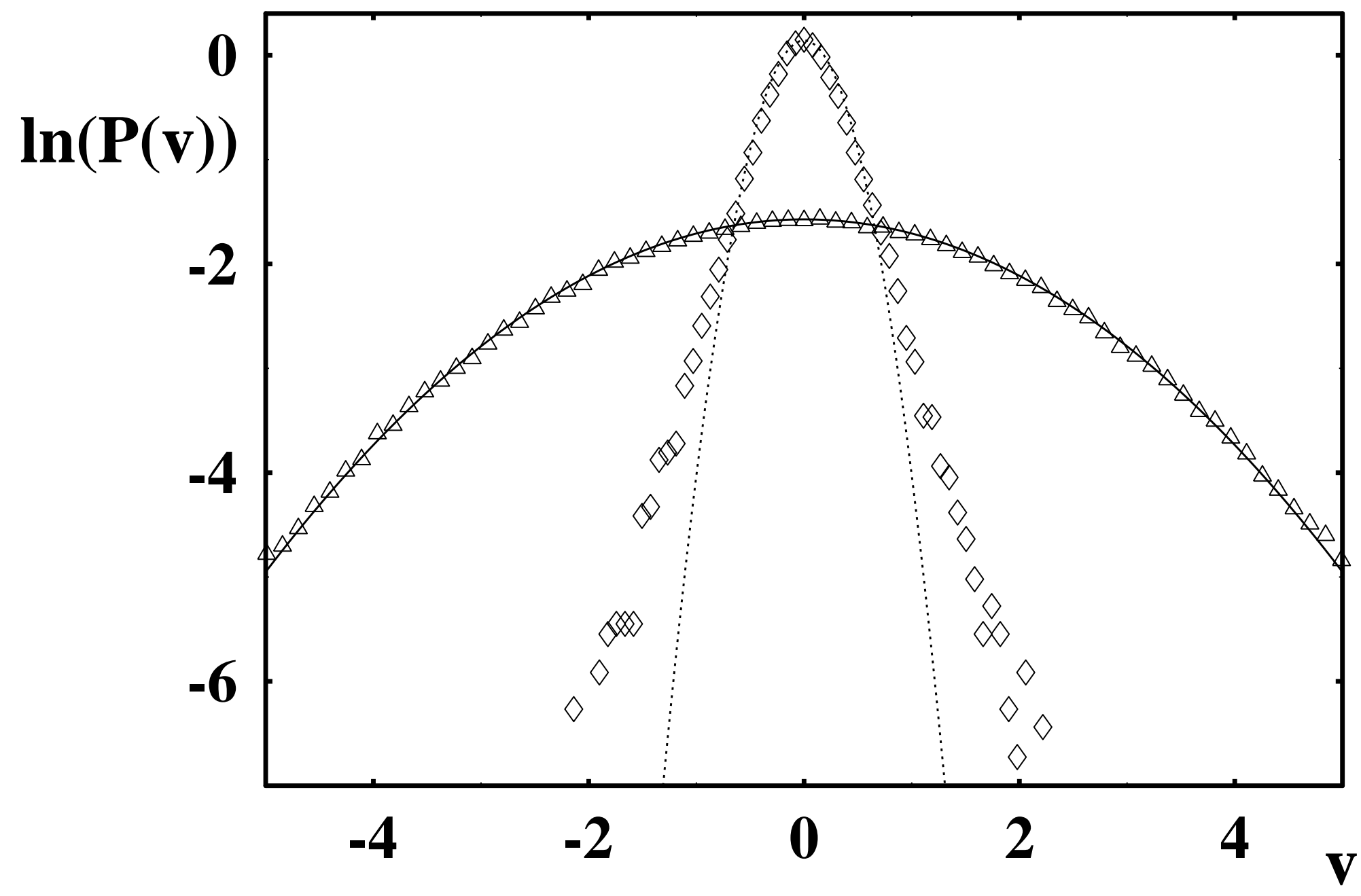

